(C) 2018, THE AUTHORS. Published by FASS Inc. and Elsevier Inc. on behalf of the American Dairy Science Association ${ }^{\circledR}$.

This is an open access article under the CC BY-NC-ND license (http://creativecommons.org/licenses/by-nc-nd/3.0/).

\title{
Prediction accuracy of direct and indirect approaches, and their relationships with prediction ability of calibration models
}

\author{
T. K. Belay, ${ }^{* 1}$ B. S. Dagnachew, ${ }^{*}$ S. A. Boison, $†$ and T. Ådnøy* \\ *Department of Animal and Aquacultural Sciences, Norwegian University of Life Sciences, PO Box 5003, 1432 Ås, Norway \\ †Nofima: The Norwegian Institute of Food, Fisheries and Aquaculture Research, Osloveien 1, 1430 Ås, Norway
}

\begin{abstract}
Milk infrared spectra are routinely used for phenotyping traits of interest through links developed between the traits and spectra. Predicted individual traits are then used in genetic analyses for estimated breeding value (EBV) or for phenotypic predictions using a single-trait mixed model; this approach is referred to as indirect prediction (IP). An alternative approach [direct prediction (DP)] is a direct genetic analysis of (a reduced dimension of) the spectra using a multitrait model to predict multivariate EBV of the spectral components and, ultimately, also to predict the univariate EBV or phenotype for the traits of interest. We simulated 3 traits under different genetic (low: 0.10 to high: 0.90) and residual (zero to high: \pm 0.90 ) correlation scenarios between the 3 traits and assumed the first trait is a linear combination of the other 2 traits. The aim was to compare the IP and DP approaches for predictions of EBV and phenotypes under the different correlation scenarios. We also evaluated relationships between performances of the 2 approaches and the accuracy of calibration equations. Moreover, the effect of using different regression coefficients estimated from simulated phenotypes $\left(\beta_{\mathrm{p}}\right)$, true breeding values $\left(\beta_{\mathrm{g}}\right)$, and residuals $\left(\beta_{\mathrm{r}}\right)$ on performance of the 2 approaches were evaluated. The simulated data contained 2,100 parents (100 sires and 2,000 cows) and 8,000 offspring (4 offspring per cow). Of the 8,000 observations, 2,000 were randomly selected and used to develop links between the first and the other 2 traits using partial least square (PLS) regression analysis. The different PLS regression coefficients, such as $\beta_{\mathrm{p}}, \beta_{\mathrm{g}}$, and $\beta_{\mathrm{r}}$, were used in subsequent predictions following the IP and DP approaches. We used BLUP analyses for the remaining 6,000 observations using the true (co)variance components that had been used for the simulation. Accuracy of prediction (of EBV and phenotype)
\end{abstract}

Received June 12, 2017

Accepted February 20, 2018.

${ }^{1}$ Corresponding author: tesfaye.kebede.belay@nmbu.no was calculated as a correlation between predicted and true values from the simulations. The results showed that accuracies of EBV prediction were higher in the DP than in the IP approach. The reverse was true for accuracy of phenotypic prediction when using $\beta_{\mathrm{p}}$ but not when using $\beta_{\mathrm{g}}$ and $\beta_{\mathrm{r}}$, where accuracy of phenotypic prediction in the DP was slightly higher than in the IP approach. Within the DP approach, accuracies of EBV when using $\beta_{\mathrm{g}}$ were higher than when using $\beta_{\mathrm{p}}$ only at the low genetic correlation scenario. However, we found no differences in EBV prediction accuracy between the $\beta_{\mathrm{p}}$ and $\beta_{\mathrm{g}}$ in the IP approach. Accuracy of the calibration models increased with an increase in genetic and residual correlations between the traits. Performance of both approaches increased with an increase in accuracy of the calibration models. In conclusion, the DP approach is a good strategy for EBV prediction but not for phenotypic prediction, where the classical PLS regression-based equations or the IP approach provided better results.

Key words: indirect prediction, direct prediction, breeding value, phenotype

\section{INTRODUCTION}

Fourier transform mid-infrared (FT-MIR) spectrometry is a potential tool for collection of data at population level for phenotypic and genetic analyses of milk components (or other derived traits). An individual's phenotype for a trait is predicted from the FT-MIR spectra. This prediction is dependent on availability of links between the trait of interest and milk spectra. The predicted trait and pedigree information and variance component estimates are used to calculate EBV and other random components included in the model based on a single-trait BLUP approach. Dagnachew et al. (2013b) referred to such an approach as indirect prediction (IP) because the multitrait spectral information is not directly used in EBV prediction procedures. Alternatively, genetic analyses can be applied directly on the milk spectral variables or on their factor scores (latent traits). The BLUP predictions of the 
random components of the model (EBV, herd test day, permanent environment, and residual) for the traits of interest are then predicted as correlated traits to the corresponding random components of the spectra. Dagnachew et al. (2013b) referred to such an approach as direct prediction (DP). Given the strong correlations among milk FT-MIR spectral variables (Soyeurt et al., 2010;Dagnachew et al., 2013a), direct genetic analyses on such correlated spectral variables may result in better accuracy of genetic evaluations (Dagnachew et al., 2013b).

The IP and DP approaches have been used to predict EBV for major milk contents (fat, protein, and lactose) in goats (Dagnachew et al., 2013b) and for traits related to fine milk compositions and technological properties of milk in cows (Bonfatti et al., 2017). Dagnachew et al. (2013b) showed that the DP approach performed better than the IP approach (i.e., relative genetic gain was improved by $3-5 \%$ in the DP compared with the IP approach) and also reported high rank correlation coefficients (0.93 to 0.96) between EBV predicted using IP and DP. However, Bonfatti et al. (2017) reported rank correlations ranging from 0.07 to 0.96 , but with $<0.5$ rank correlations for most traits investigated in their study. Belay et al. (2017) adopted the 2 approaches to predict phenotype for BHB in blood from milk spectra and reported a slightly better phenotypic prediction by the IP than the DP approach.

Based on studies done so far, it is difficult to make a conclusive remark on whether the DP approach is better than the IP approach for EBV or phenotype prediction. Each of the studies cited in the preceding paragraph has their limitation. For example, independent chemical analyses (reference values) for the milk contents were not available in the study of Dagnachew et al. (2013b; i.e., the study used phenotypes predicted from the same spectra as reference values for both model calibration and evaluation). Possibly for the reason above, the coefficients of determination $\left(\mathbf{R}^{2}\right)$ were very high $(>0.96)$. Moreover, the accuracies of EBV were estimated based on coefficient matrices of the mixed model equations in Dagnachew et al. (2013b). In the study of Bonfatti et al. (2017), reference values measured independently of the spectra were used to develop prediction equations that had medium (0.35) to high (0.86) $\mathrm{R}^{2}$ values; however, it is difficult to distinguish the approaches that performed better based on that study because the IP and DP approaches were evaluated based on rank correlations. In an attempt to predict phenotypes with IP and DP (Belay et al., 2017), the $\mathrm{R}^{2}$ were low and data sets used for the model validation as well as for evaluation of the 2 approaches were small.

Furthermore, in the 3 studies, covariance components of the latent traits estimated by the DP approach were converted to variance components to be used in the IP approach using links (regression coefficients) estimated based on phenotypes $\left(\boldsymbol{\beta}_{\mathrm{p}}\right)$. Similarly, EBV of the latent traits were converted into single-trait EBV using phenotype based links. Utilization of a partial least square (PLS) regression coefficient estimated from phenotypes (i.e., $\beta_{\mathrm{p}}$ to convert EBV of latent traits into EBV of trait of interest) does not seem appropriate; this might have an effect on the performance of the approaches. Parameters estimated at one level (e.g., at phenotypic level) were used at another level (e.g., at genetic level); therefore, the effect of using appropriate conversion parameters [e.g., estimated from true breeding values $\left(\boldsymbol{\beta}_{\mathrm{g}}\right)$ ] to convert multitrait structures to single-trait structures on performances of the 2 approaches is unknown and needs to be studied. Moreover, relationship between performance of the 2 approaches and accuracy of calibration models is unclear.

Therefore, objectives of our study were (1) to evaluate performance of the IP and DP approaches for prediction of EBV and phenotype under different genetic and residual structures between traits; (2) to evaluate effect of using different PLS regression coefficients (e.g., $\beta_{\mathrm{p}}$, $\beta_{\mathrm{g}}$, and so on) for converting covariance components or EBV of latent traits into univariate structure on performance of the 2 approaches; and (3) to study the relationship between performance of the 2 approaches and accuracy of calibration models.

\section{MATERIALS AND METHODS}

\section{Simulation}

A simulation program written in $\mathrm{R}$ ( $\mathrm{R}$ Core Team, 2016) to make single- and multitrait data sets based on pedigree was used. The $\mathrm{R}$ codes used for the simulation can be found online (https://github.com/soloboan/ Multi-trait_simulations). A base population consisting of 100 sires and 2,000 cows with 3 traits under different genetic and residual correlation scenarios were simulated. Subsequently, 2 generations of data were simulated, with 2,100 parents (100 sires and 2,000 cows) in each generation. It was assumed that a cow would have 4 offspring per generation, resulting in 8,000 offspring per generation, from which parents for the next generation were selected. Sex ratio of offspring was fixed at $50 \%$. Parents were randomly selected and the selected animals were randomly mated by random union of gametes leading to pseudo-overlapping generations as is mostly used in cattle breeding.

Variance components and the corresponding heritabilities used for simulation of the 3 traits are given in Table 1, whereas the different genetic and residual correlation scenarios are presented in Table 2. The 
Table 1. Overall means, genetic and residual variances, and heritabilities of the simulated traits ${ }^{1}$

\begin{tabular}{lcccc}
\hline Trait & Mean & Genetic variance & Residual variance & Heritability \\
\hline Trait 1 & 3.450 & 0.027 & 0.044 & 0.377 \\
Trait 2 & -0.030 & 0.089 & 0.839 & 0.096 \\
Trait 3 & -0.050 & 0.158 & 0.686 & 0.187 \\
\hline
\end{tabular}

${ }^{1}$ Except residual variance, all other parameters were estimates from previous studies (estimates for trait 1 that represent protein percent are from T. K. Belay, unpublished data), whereas estimates for the other 2 traits (trait 2 and 3) represent latent traits derived from milk spectra in Belay et al. (2015). Residual variance was calculated given the heritability and genetic variance of each trait.

first trait (hereafter referred to as the focal trait) was assumed to be milk protein content, and is a linear combination of the 2 other traits, which were assumed to be latent traits earlier derived from spectral variables. Mean, genetic variance, and heritability of the focal trait were taken from a previous study (T. K. Belay, unpublished data), whereas those of the other 2 traits were based on estimates already reported (Belay et al., 2015). Residual variances for the 3 traits were calculated from the genetic variance and heritability of each trait. Twelve possible pairs of genetic (3 scenarios) and residual (4 scenarios) correlation scenarios were simulated. The genetic correlations between the 3 traits were assumed to be either low (0.10 to 0.25 ), medium (0.50 to 0.70$)$, or high (0.80 to 0.90$)$ whereas the residual correlations were grouped as zero, low, medium and high. Simulations were replicated 100 times for each scenario. The simulated data consisted of generation number, pedigree, sex, true breeding values (TBV), residuals, and true phenotype values (TPV) for each trait.

\section{Multivariate Calibration Models}

The link between the focal trait (milk protein content) and the other 2 traits were developed by PLS regression using the pls package (Mevik and Wehrens, 2007) implemented in $\mathrm{R}$ ( $\mathrm{R}$ Core Team, 2016). The PLS regression analyses were done on 2,000 observations randomly sampled without replacement from the
8,000 offspring population in the second generation. The calibrations were done for each replication within a scenario and average values of calibration outputs reported. The PLS regression analysis was undertaken using phenotypes (TBV + errors), as well as the TBV and residuals of the focal trait as dependent variables. In these analyses, the 2 other traits were used as predictor variables. The PLS regression coefficients $(\beta)$ from phenotype $\left[\beta\right.$-phenotypic $\left.\left(\beta_{\mathrm{p}}\right)\right]$, TBV $\left[\beta\right.$-genetic $\left.\left(\beta_{\mathrm{g}}\right)\right]$, and error $\left[\beta\right.$-residual $\left.\left(\boldsymbol{\beta}_{\mathbf{r}}\right)\right]$ were used to convert multivariate structures into univariate in the subsequent predictions or calculations. Prediction of EBV and phenotypes were performed following the DP or IP approaches. The PLS $\mathrm{R}^{2}$ for phenotype $\left(\mathrm{R}_{\mathrm{p}}{ }^{2}\right)$, and TBV $\left(\mathrm{R}_{\mathrm{g}}{ }^{2}\right)$ were plotted against prediction accuracy of IP and DP approaches to evaluate relationship between accuracy of calibration model and the 2 approaches. Figure 1 shows a schematic representation of the 2 prediction approaches.

\section{Direct Prediction}

In this approach, procedures were similar to those described in previous studies for prediction of EBV (Dagnachew et al., 2013b; Bonfatti et al., 2017) or phenotypes (Belay et al., 2017). In the current study, however, the steps for dimension reduction of spectral information into few latent variables and variance component estimation for those latent variables were bypassed. Trait 2 and 3 in our simulations were assumed

Table 2. Scenarios for genetic and residual correlations between the 3 traits used in simulations

\begin{tabular}{|c|c|c|c|c|c|c|c|}
\hline \multirow[b]{3}{*}{ Item $^{1}$} & \multicolumn{7}{|c|}{ Scenario } \\
\hline & \multicolumn{3}{|c|}{ Genetic } & \multicolumn{4}{|c|}{ Residual } \\
\hline & High & Medium & Low & High & Medium & Low & Zero \\
\hline$\overline{\mathrm{r}_{12}}$ & 0.80 & 0.50 & 0.25 & -0.80 & -0.50 & -0.25 & 0 \\
\hline $\mathrm{r}_{13}$ & 0.90 & 0.70 & 0.10 & 0.90 & 0.70 & 0.10 & 0 \\
\hline $\mathrm{r}_{23}$ & 0.85 & 0.65 & 0.15 & -0.85 & -0.60 & -0.15 & 0 \\
\hline
\end{tabular}

${ }^{1} \mathrm{r}_{12}, \mathrm{r}_{13}$, and $\mathrm{r}_{23}$ are correlations between traits 1 and 2,1 and 3 , and 2 and 3 , respectively. Trait 1 is the focal trait (protein percent) and traits 2 and 3 are assumed to be latent traits after dimensionality reduction of spectra information. 
to be the latent variables obtained after dimensional reduction of spectral information. The genetic variances and heritabilities used in simulating trait 2 and 3 were based on our previous study (Belay et al., 2015). The BLUP estimates (e.g., EBV) for trait 2 and 3 were obtained by fitting bivariate animal models using Wombat (Meyer, 2007). True (co)variance components were used to predict EBV and other model components including predicted values for trait 2 and 3 . The model in matrix notation was

$$
\mathbf{t}=\mathbf{X b}+\mathbf{Z a}+\mathbf{e},
$$

where $\mathbf{t}$ is a vector of simulated phenotypes of trait 2 and $3 ; \mathbf{b}$ is a mean (fixed effect); $\mathbf{a}$ is a vector of random additive genetic effects; $\mathbf{e}$ is a vector of random

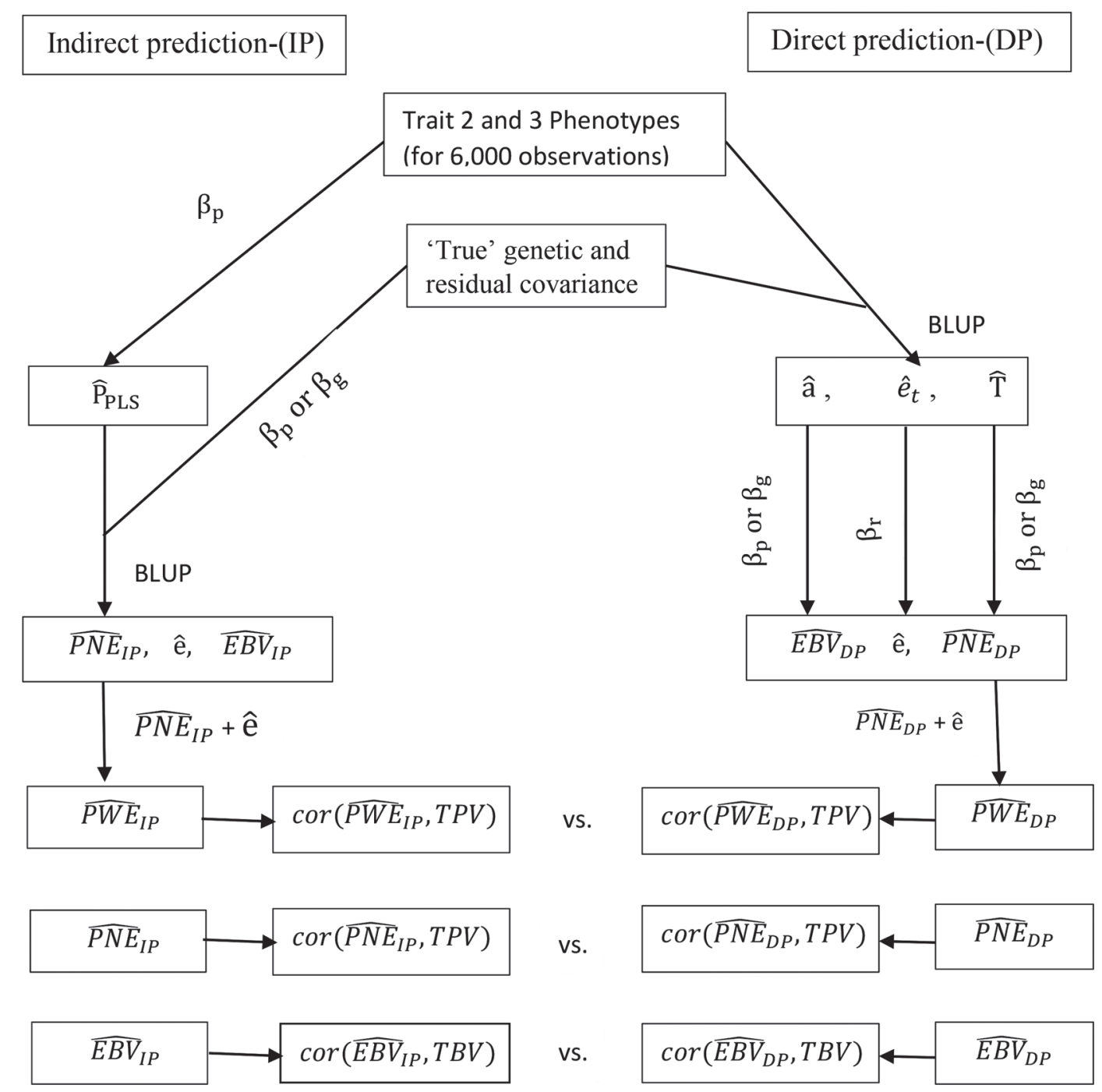

Figure 1. Schematic representation of the indirect (IP) and direct (DP) prediction approaches. For the IP method, phenotype for focal trait was predicted $\left(\widehat{P}_{P L S}\right)$ from traits 2 and 3 using regression coefficient $\left(\beta_{\mathrm{p}}\right)$ estimated from true phenotype values (TPV). A BLUP was then applied to this predicted trait using the true genetic and residual covariance of traits 2 and 3 after converting into respective variances through $\beta_{\mathrm{p}}$ or $\beta_{\mathrm{g}}$ [regression coefficient from true breeding values (TBV)] for EBV $\left(\widehat{E B V}_{I P}\right)$ and phenotypic $\left(\widehat{P N E}_{I P}\right)$ prediction. The $\widehat{P N E}_{I P}$ obtained directly from BLUP do not contain residual effects ( $)$ ) whose addition to the $\widehat{P N E}_{I P}$ resulted in phenotype that contained error $\left(\widehat{P W E}_{I P}\right)$. For the DP method, traits 2 and 3 were analyzed multivariately with a genetic model before predicted model components [such as genetic ( $\hat{a})$ and re$\operatorname{sidual}\left(\hat{\mathrm{e}}_{t}\right)$ and phenotype $(\hat{\mathrm{T}})$ predicted for trait 2 and 3$]$ are combined through the $\beta_{\mathrm{p}}$ or $\beta_{\mathrm{g}}$ to eventually predict phenotype $\left(\widehat{P N E}_{D P}\right)$ and $\mathrm{EBV} \mathrm{V}_{\mathrm{DP}}$ of focal trait. Residual part of predicted traits 2 and $3\left(\hat{e}_{t}\right)$ was converted into single-trait residual (ê) through the $\beta_{\mathrm{r}}$ (regression coefficient estimated from the residual part of simulated phenotypes) and the ê added to the $\widehat{P N E}_{D P}$ to obtain predicted phenotype that contained residual effects $\left(\widehat{P W E}_{D P}\right)$. 
residual effects; $\mathbf{X}$ is a column of ones and $\mathbf{Z}$ is a design matrix that relates records to the corresponding effects.

The following (co)variance structure for trait 2 and 3 was assumed:

$$
\operatorname{var}\left[\begin{array}{l}
\mathbf{a} \\
\mathbf{e}
\end{array}\right]=\left[\begin{array}{cc}
\mathbf{G} \otimes \mathbf{A} & 0 \\
0 & \mathbf{R} \otimes \mathbf{I}
\end{array}\right],
$$

where $\mathbf{G}$ is genetic (co)variance matrix for trait 2 and 3 , and $\mathbf{R}$ is the residual (co)variance matrix. All (co) variance matrices were $2 \times 2$. I and $\mathbf{A}$ are identity and additive relationship matrices, respectively, and $\otimes$ is the Kronecker product.

$E B V$ of Focal Trait from $E B V$ of Trait 2 and 3. The predicted EBV $\left(\hat{a}_{2 \times 2}\right)$ of trait 2 and 3 were directly transformed into $\mathrm{EBV}$ of focal trait $\left(\widehat{E B V}_{D P}\right)$ through either $\beta_{\mathrm{p}}$ or $\beta_{\mathrm{g}}$ (i.e., $\widehat{E B V}_{D P}=\hat{a}_{2 \times 2} \hat{\beta}_{i(2 \times 1)}$, where $i=\beta_{\mathrm{p}}$ or $i=\beta_{\mathrm{g}}$ ).

Phenotypes for Focal Trait from Predicted Trait 2 and 3. In addition to prediction of the random effects $\left(\hat{a}_{2 \times 2}\right.$ and $\left.\hat{e}_{\mathrm{n} \times 2}\right)$, predicted phenotypes for trait 2 and $3\left(\widehat{\mathrm{T}}_{\mathrm{n} \times 2}\right)$ were also computed in the BLUP analysis. Predicted phenotypes not adding the residual term $\left(\hat{e}_{\mathrm{n} \times 2}\right)$ for the focal trait $\left(\widehat{P N E}_{D P}\right)$ were computed from the predicted phenotypes of trait 2 and $3\left(\widehat{\mathrm{T}}_{\mathrm{n} \times 2}\right)$. The

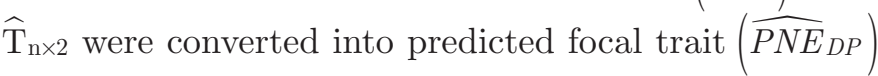
either through $\beta_{\mathrm{p}}$ or $\beta_{\mathrm{g}}$. Mathematically $\widehat{P N E}_{D P}=\widehat{\mathrm{T}}_{\mathrm{n} \times 2} \widehat{\hat{\beta}}_{i(2 \times 1)}$, where $i$ is as defined above and $\mathrm{n}$ is number of observations. This predicted focal trait $\widehat{P N E}_{D P}$ did not contain residual effects $\left(\hat{e}_{\mathrm{n} \times 2}\right)$. Alternatively, the residual effects $\left(\hat{e}_{\mathrm{n} \times 2}\right)$ were transformed into univariate form through $\beta_{\mathrm{r}}$ and then added to the $\widehat{P N E}_{D P}$ to obtain phenotypes that contained residual effects $\quad\left(\widehat{P W E}_{D P}\right)$. Mathematically $\widehat{P W E}_{D P}=\widehat{\mathrm{T}}_{\mathrm{n} \times 2} \hat{\beta}_{i(2 \times 1)}+\hat{e}_{\mathrm{n} \times 2} \hat{\beta}_{\mathrm{r}(2 \times 1)}$.

\section{Indirect Prediction}

In this approach, the focal trait was predicted from the other 2 simulated traits by classical PLS regression using the $\beta_{\mathrm{p}}$ estimated above $\left(\widehat{P}_{P L S}=T P V_{\mathrm{n} \times 2} \widehat{\beta}_{\mathrm{p}(2 \times 1)}\right)$, and then genetic analysis (BLUP) was conducted on the predicted phenotypes $\left(\widehat{P}_{P L S}\right)$. The true (co)variance components (those used in the simulation) of the 2 other traits were converted into variance components through $\beta_{\mathrm{p}} \quad$ or $\beta_{\mathrm{g}} \quad\left(\sigma_{a}^{2}={\widehat{\beta^{\prime}}}_{i(1 \times 2)} \mathbf{G}_{2 \times 2} \widehat{\beta}_{i(2 \times 1)} \quad\right.$ and $\left.\sigma_{e}^{2}={\widehat{\beta^{\prime}}}_{i(1 \times 2)} \mathbf{R}_{2 \times 2} \widehat{\beta}_{i(2 \times 1)}\right)$, where $i$ is as defined above, $\sigma_{a}^{2}$ is additive genetic variance, and $\sigma_{e}^{2}$ is residual variance. These variance components were then used in singletrait BLUP analysis. The same animal models as in the DP were fitted, but with a single trait. In this case, we assumed $\operatorname{var}(a)=\mathbf{A} \sigma_{a}^{2}$, and $\operatorname{var}(e)=\mathbf{I} \sigma_{e}^{2}$.

Predicted focal trait without residual effects $\left(\widehat{P N E}_{I P}\right)$ and $\mathrm{EBV}\left(\widehat{E B V}_{I P}\right)$ and solutions for random residuals $(\hat{e})$ were directly obtained from the BLUP analysis. The BLUP analysis of PLS-predicted traits $\left(\widehat{P}_{P L S}\right)$ for prediction of itself (the same phenotype) may be superfluous, but was done to conform to the phenotype predicted in the DP $\left(\widehat{P N E}_{D P}\right)$. Similar to the DP, the residual effects $(\hat{e})$ from BLUP were added to the $\widehat{P N E}_{I P}$ to obtain a phenotype that contained error (i.e., $\left.\widehat{P W E}_{I P}=\widehat{P N E}_{D P}+\hat{e}\right)$. Thus, in addition to the $\widehat{P}_{P L S}$, we got 2 vectors of the predicted focal trait under the IP approach, $\widehat{P N E}_{I P}$ and $\widehat{P W E}_{I P}$.

\section{Evaluation of the IP and DP Approaches}

Performance of the 2 approaches were evaluated based on accuracy of EBV or phenotype prediction. Accuracy of EBV prediction was defined as the correlation between TBV and EBV. Pearson correlation coefficients between DP predicted EBV and TBV of the focal trait were computed and compared with correlation between the IP-predicted EBV and TBV. In a similar manner, accuracy of phenotypic prediction was defined as the correlation between predicted phenotypes and simulated phenotypes. The predicted phenotypes, such as $\widehat{P N E}_{D P}$ and $\widehat{P W E}_{D P}$ from the DP approach, $\widehat{P N E}_{I P}$ and $\widehat{P W E}_{I P}$ from the IP approach, and $\widehat{P}_{P L S}$ from classical PLS, are different predictions of the same trait (e.g., milk protein content).

\section{RESULTS AND DISCUSSION}

\section{Accuracy of EBV Prediction Under the DP and IP Approaches}

Accuracy of EBV prediction for sires as well as for all animals with records were computed. However, only sire evaluations are presented here because the trends for the IP and DP approaches were similar. Accuracy of sire EBV predicted using the DP and IP approaches are presented in Table 3. In the DP approach, EBV of trait 2 and 3 were converted into EBV of the focal trait (milk protein content) using PLS regression coefficients estimated based on TBV $\left(\beta_{\mathrm{g}}\right)$ or TPV $\left(\beta_{\mathrm{p}}\right)$. In the IP 
Table 3. Mean accuracy ${ }^{1}$ (SE) of predicted breeding values of sires using the direct (DP) and indirect (IP) prediction approaches under different genetic and residual correlation scenarios using regression coefficients estimated based on true breeding values $\left(\beta_{\mathrm{g}}\right)$ and true phenotype $\left(\beta_{\mathrm{p}}\right)$

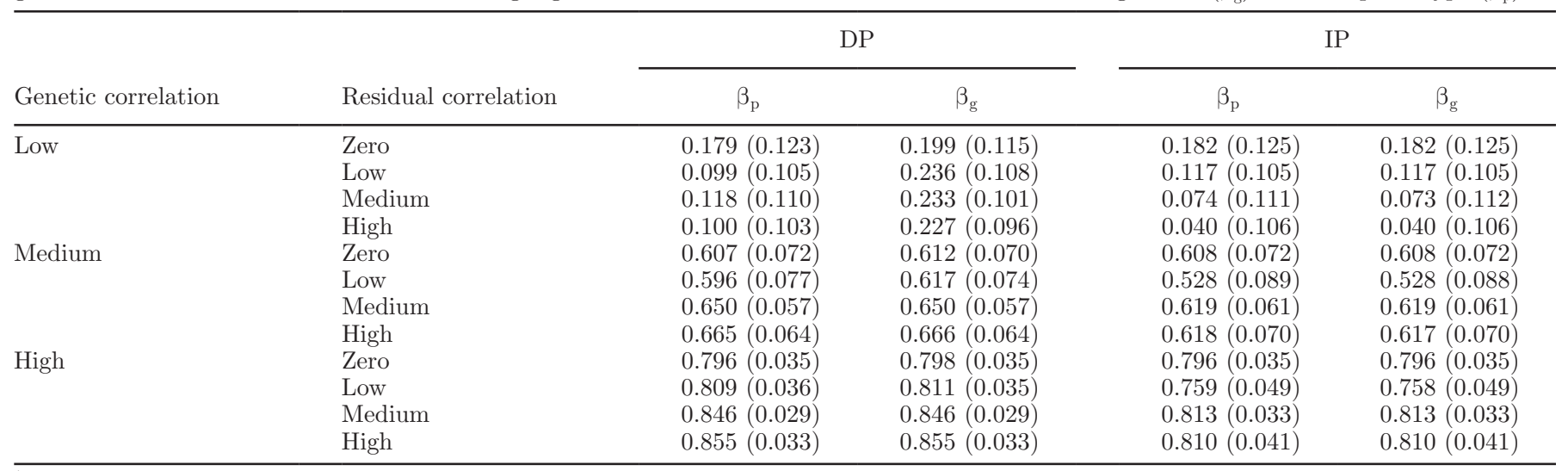

$\overline{{ }^{1} \text { Accuracy was defined as a correlation between predicted EBV and simulated true breeding values (TBV). Average of } 100 \text { replicates per scenario }}$ is reported and the SE were calculated as the SD of the 100 accuracies for each scenario.

approach, the covariance components of trait 2 and 3 were converted into variance of the focal trait using the $\beta_{\mathrm{g}}$ or $\beta_{\mathrm{p}}$. Those variances were used in univariate BLUP analyses for EBV or phenotype prediction. In both DP and IP approaches, the accuracy of sire EBV predictions were significantly $(P=0.0)$ increased as the genetic correlations between all the traits were increased regardless of residual correlations. At a given genetic correlation scenario, EBV accuracy also (significantly, $P<0.05)$ increased with an increase in residual correlation, except at low genetic correlation scenarios where no clear trend was observed. Increases in residual correlations should not increase accuracy of EBV except that residual correlations between traits could lead to artificial resemblance between EBV because the phenotypes are correlated.

In all possible combinations of genetic and residual correlation scenarios, regardless of the types of $\beta$ used, accuracies of EBV prediction using the DP approach were significantly $(P<0.01)$ higher than the corresponding predictions in the IP approach. The exception was at zero residual correlations, where we found no significant $(P>0.05)$ difference in accuracy between the 2 approaches (Table 3 ). In agreement with the current study, Dagnachew et al. (2013b) reported a reduction in prediction error variance (and hence an increase in accuracy) using the DP approach instead of the IP approach. We also evaluated performance of the 2 approaches when little to no correlation between trait 2 and 3 was observed, but with varying correlations between the focal trait and the other 2 traits, and found similar EBV accuracy prediction in both IP and DP approaches (results not shown). This indicates some correlations should be present between the other 2 traits, in addition to the correlations to the focal trait for the DP performing better than the IP approach. This is because the DP approach uses the covariance structure between the other 2 traits to predict EBV for the focal trait. If no covariance exists between the other 2 traits, no mathematical explanation exists to expect better performance for the DP than the IP approach. Therefore, for better EBV accuracy prediction in the DP approach, existence of correlations between focal trait and the other 2 traits as well as between the other 2 traits are a prerequisite.

Comparison between the effects of using $\beta_{\mathrm{p}}$ and $\beta_{\mathrm{g}}$ on accuracy of EBV were made within each approach. In the DP approach, we found significantly $(P=0)$ higher accuracy of EBV when using $\beta_{\mathrm{g}}$ rather than $\beta_{\mathrm{p}}$ only in the low genetic correlation scenario. The exception was in the low genetic-zero residual correlation scenario, where we noted no significant difference in EBV accuracy between using $\beta_{\mathrm{p}}$ and $\beta_{\mathrm{g}}$. This suggested that if the genetic correlations between the 3 traits are low, an appropriate regression coefficient (e.g., $\beta_{\mathrm{g}}$ ) should be used for better EBV accuracy in the DP approach. However, we observed no significant $(P>0.05)$ difference in EBV accuracy between using the $\beta_{\mathrm{p}}$ and $\beta_{\mathrm{g}}$ in the IP approach; this is interesting, as the IP approach is the commonly used method in genetic analyses of traits predicted from milk FT-MIR spectra. It might be difficult to estimate $\beta_{\mathrm{g}}$ from real data, so that it is a challenge. In a real data setting, an estimate of the genetic regression coefficient $\left(\hat{\beta}_{g}\right)$ can be obtained through a series of steps. First, we need to estimate variance components and predict breeding values for the other 2 traits (or for each of the spectra variables). This can be done individually for each spectral wavenumber by fitting single-trait animal model (Bittante and Cecchina- 
to, 2013; Wang et al., 2016) or for latent traits that derived from spectra via multitrait genetic analysis and then back-transforming EBV of the latent traits to spectral scale (Soyeurt et al., 2010; Dagnachew et al., 2013b;Bonfatti et al., 2017). Genetic regression coefficient can then be computed by regressing the phenotype of the focal trait on EBV of each spectral variables or latent traits (see Appendix for detailed description). The $\hat{\beta}_{\mathrm{g}}$ estimated from real data would approximate the $\beta_{g}$, and performances of the IP and DP approaches under $\hat{\beta}_{\mathrm{g}}$ are expected to be in line with their performances under $\beta_{\mathrm{g}}$. However, this dependents on how accurately $\hat{\beta}_{\mathrm{g}}$ will be estimated (i.e., how $\hat{\beta}_{\mathrm{g}}$ is a best estimator of $\beta_{\mathrm{g}}$ ).

In the current study, which depended on simulated data, and a previous study, which used real data (Dagnachew et al., 2013b), the DP approach improved accuracy of EBV prediction. In the DP approach, EBV are predicted once for latent variables derived from spectra and later combined into EBV of focal traits without needing to first predict phenotypes for the focal traits from milk spectra. This is particularly important when FT-MIR calibration equations are available for a high number of traits (Bonfatti et al., 2017). Such use of the DP approach for spectra may be possible when the spectral dimension is reduced by principal component analysis (PCA) and not as feasible with PLS. This is because parameter estimates for the latent variables from PCA are population parameters that characterize any information available in the milk spectra. This is not the case, for example, for factor scores from the PLS regression, as they mainly contain information related to the particular trait used in the calibration. However, the retained latent variables from PCA might not contain all information about the focal trait (Soyeurt et al., 2010; Dagnachew et al., 2013b; Bonfatti et al., 2017).

\section{Relationships Between Accuracy of EBV Prediction and Calibration Equations}

Coefficient of determinations in calibration models estimated from simulated phenotypes $\left(\mathrm{R}_{\mathrm{p}}{ }^{2}\right)$ ranged from 0.004 (for low genetic and low residual correlation scenario) to 0.787 (for high genetic and high residual correlation scenario). The $\mathrm{R}_{\mathrm{p}}{ }^{2}$ increased with the increase in either genetic or residual or both correlations (e.g., Figure 2). The corresponding estimates from true breeding values $\left(\mathrm{R}_{\mathrm{g}}{ }^{2}\right)$ ranged from 0.070 (for low genetic correlation) to 0.814 (for high genetic correlation). The $\mathrm{R}_{\mathrm{g}}{ }^{2}$ increased with increase in genetic correlations, but did not change as residual correlations increased (e.g., Figure 3). At most of the correlation scenarios considered, estimates for the $\mathrm{R}_{\mathrm{g}}{ }^{2}$ were higher than the corresponding values for the $\mathrm{R}_{\mathrm{p}}^{2}$.

The relationships between performance of the IP and DP approaches in predicting individual EBV and predictive ability of calibration models $\left(\mathrm{R}^{2}\right)$ are depicted in Figure 2 for $\beta_{\mathrm{p}}$ and $\mathrm{R}_{\mathrm{p}}{ }^{2}$ and in Figure 3 for $\beta_{\mathrm{g}}$ and $\mathrm{R}_{\mathrm{g}}{ }^{2}$. When using the $\beta_{\mathrm{p}}$, prediction accuracy of the IP and DP approaches increased with increase in predictive ability of the calibration models $\left(\mathrm{R}_{\mathrm{p}}{ }^{2}\right)$. The exception was at low genetic correlation (with zero to high residual correlation scenarios), where EBV accuracy generally decreased as the $\mathrm{R}_{\mathrm{p}}{ }^{2}$ increased. This indicated that improvement in residual structure for lowly genetically correlated traits has no contribution in improving their EBV prediction accuracy. In other words, calibration models with higher $\mathrm{R}^{2}$ do not necessarily result in better EBV prediction accuracies when genetic correlations between traits are low. What really matters for better accuracy of EBV prediction is the genetic correlation compared with residual correlation. For example, at zero (Figure 2a) or low (Figure 2b) residual structure, $\mathrm{R}_{\mathrm{p}}{ }^{2}$ of the calibration models slightly increased with an increase in genetic correlations (low to high), but EBV accuracy greatly improved compared with the $\mathrm{R}_{\mathrm{p}}{ }^{2}$. At high residual correlation (Figure 2d) as well as at low residual correlation (Figure 2b) with medium to high genetic correlation scenarios, the difference in prediction accuracy between the IP and DP approaches were more visible $(P<0.05)$ than at either zero (Figure 2a) or medium (Figure 2c) residual correlations. This was clearer for EBV of all animals with records than for EBV of sires; that is, as the predictive ability of the calibration models increased, the difference in prediction accuracy between the IP and DP approaches became more apparent (results not shown).

Similar to when using the $\beta_{\mathrm{p}}$, EBV prediction accuracy of the IP and DP approaches in using the $\beta_{\mathrm{g}}$ increased with increase in predictive ability $\left(\mathrm{R}_{\mathrm{g}}{ }^{2}\right)$ of the calibration equations (Figure 3 ). As expected, the residual structure (Figure 3a-d) in this case had no effect on predictive ability of the calibration models but on accuracy of EBV prediction, as the predicted EBV was derived from a phenotype that contained both residual and genetic information. Increases in genetic correlations between traits increased both accuracy of the 2 approaches (IP and DP) and predictive ability of the calibration models regardless of the residual structures. Except at zero residual correlation (Figure 3a), the difference in performance of the 2 approaches became visible $(P<0.05)$ as the residual correlations increased (Figure $3 \mathrm{~b}-\mathrm{d}$ ), especially at low genetic scenarios. However, such clear differences were not observed when 


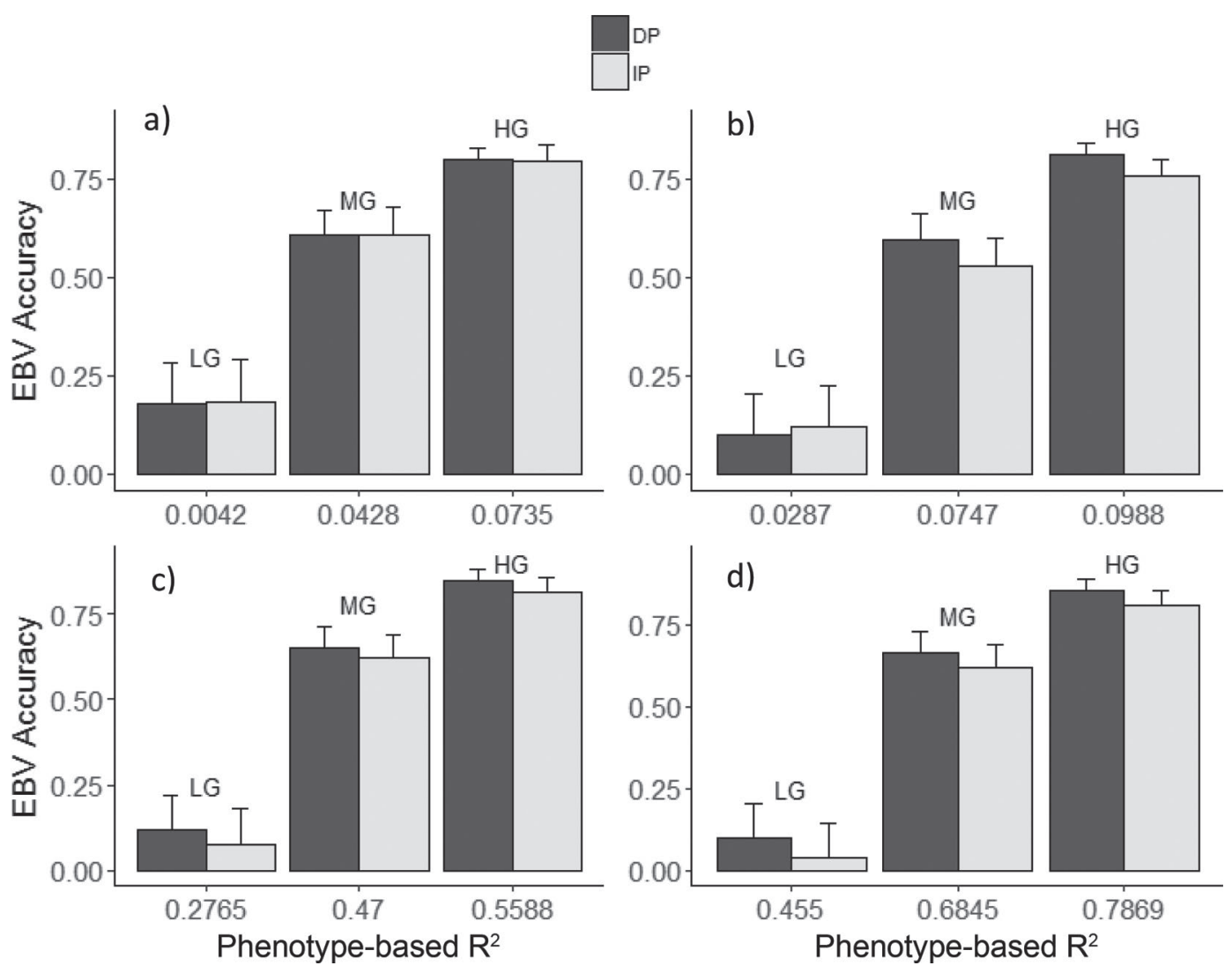

Figure 2. Determination coefficients of calibration models estimated based on phenotype and mean EBV accuracy of sire predicted using the direct (DP) and indirect (IP) prediction approaches. The mean EBV accuracy was predicted under low (LG), medium (MG), and high (HG) genetic correlations with zero (a), low (b), medium (c), and high (d) residual correlation scenarios. The regression coefficient was used to convert covariance components of traits 2 and 3 into variance components in IP or EBV of traits 2 and 3 into EBV of the focal trait in DP. The error bars represent the SE of prediction.

using $\beta_{\mathrm{p}}$ (Figure 2), indicating that performance of the DP approach is sensitive to the type of PLS regression coefficients used.

The significantly $(P=0)$ better performance of DP when using $\beta_{\mathrm{g}}$, especially at low genetic correlation might be due to the simultaneous increase in genetic correlations between the focal trait and the other 2 traits (trait 2 and 3 ) as well as between trait 2 and 3 . As the genetic correlation between the traits increases, their information content becomes similar (i.e., one trait provides more information about the other traits); hence, simultaneous analysis of such traits would be slightly different from analyzing them separately. The significantly $(P=0)$ better performance of DP in using $\beta_{\mathrm{g}}$ at low genetic correlation scenario might also be related to predictive ability of calibration models from which $\beta_{\mathrm{g}}$ was estimated. At low genetic correlation, predictive ability of calibration models was low $(<0.10)$ and $\beta_{\mathrm{g}}$ captures little information about the intended trait. Hence, EBV of trait 2 and 3 were predicted more accurately due to utilization of covariance between them (DP). However, the corresponding EBV prediction in IP was relatively inaccurate, as the phenotypes from which the EBV were derived were poorly predicted through a $\beta_{\mathrm{g}}$ that contained little information. At high predictive ability of calibration models, the IP approach gives accurate prediction and not much gain from the DP that uses little extra information not used by the IP approach, resulting in small difference in performance between the approaches. That means performance of the IP approach is approaching that of the DP approach as accuracy of the calibration models increased.

In our previous study, we suggested that predictive ability of calibration model could affect performance of the 2 approaches. The suggestion was made based on the work of Dagnachew et al. (2013b), where high $\mathrm{R}^{2}$ was associated with better performance in DP than in IP, and Belay et al. (2017), where low $\mathrm{R}^{2}$ was associated with inferior performance in the DP compared with the 
IP approach. In the study of Dagnachew et al. (2013b), accuracies of EBV prediction were estimated based on prediction error variance, but Belay et al. (2017) estimates were based on correlation between observed and predicted phenotype. However, Bonfatti et al. (2017) reported absence of relationships between rank correlations (between EBV obtained by the IP and the DP approach) and predictive ability of calibration equations. In the current study, we have shown that performance of the 2 approaches increased with an increase in the predictive ability of calibration models, but it is not necessary to have calibration equation with high $\mathrm{R}^{2}$ for the DP approach to perform better the IP approach for EBV prediction. This might not be the case for phenotypic prediction (details in next sections).

\section{Accuracy of Phenotypic Prediction $\left(\widehat{P N E}_{I P}\right.$ and $\left.\widehat{P N E}_{D P}\right)$}

Similar to accuracies of EBV, accuracies of predicted phenotypes generally increased with an increase in cor- relations (genetic and residual) between the focal trait and the other 2 traits. However, no clear trend was observed for DP compared with IP performance when the residual correlation increased for the low genetic scenario when using $\beta_{\mathrm{g}}$ (Table 4 ). When using $\beta_{\mathrm{p}}$, accuracy of predicted phenotypes in IP approach were higher (or significantly $(P<0.001)$ higher at low to high genetic correlations with medium or high residual correlation scenarios) than the corresponding estimates in the DP approach. This is in agreement with our previous study (Belay et al., 2017), where slightly better prediction of blood BHB was found in the IP than in the DP approach; however, it is in contrast with EBV accuracy observed in the current (Table 3 or Figures 2 and 3) and previous (Dagnachew et al., 2013b) studies. When using $\beta_{g}$, accuracies of predicted phenotypes in the IP approach were also significantly $(P<0.05)$ higher than the corresponding estimates in the DP approach. The exception was at zero residual correlation with low to high genetic correlation scenarios, where we found no significant $(P>0.05)$ difference between the DP and IP approaches. For the DP approach, we noted

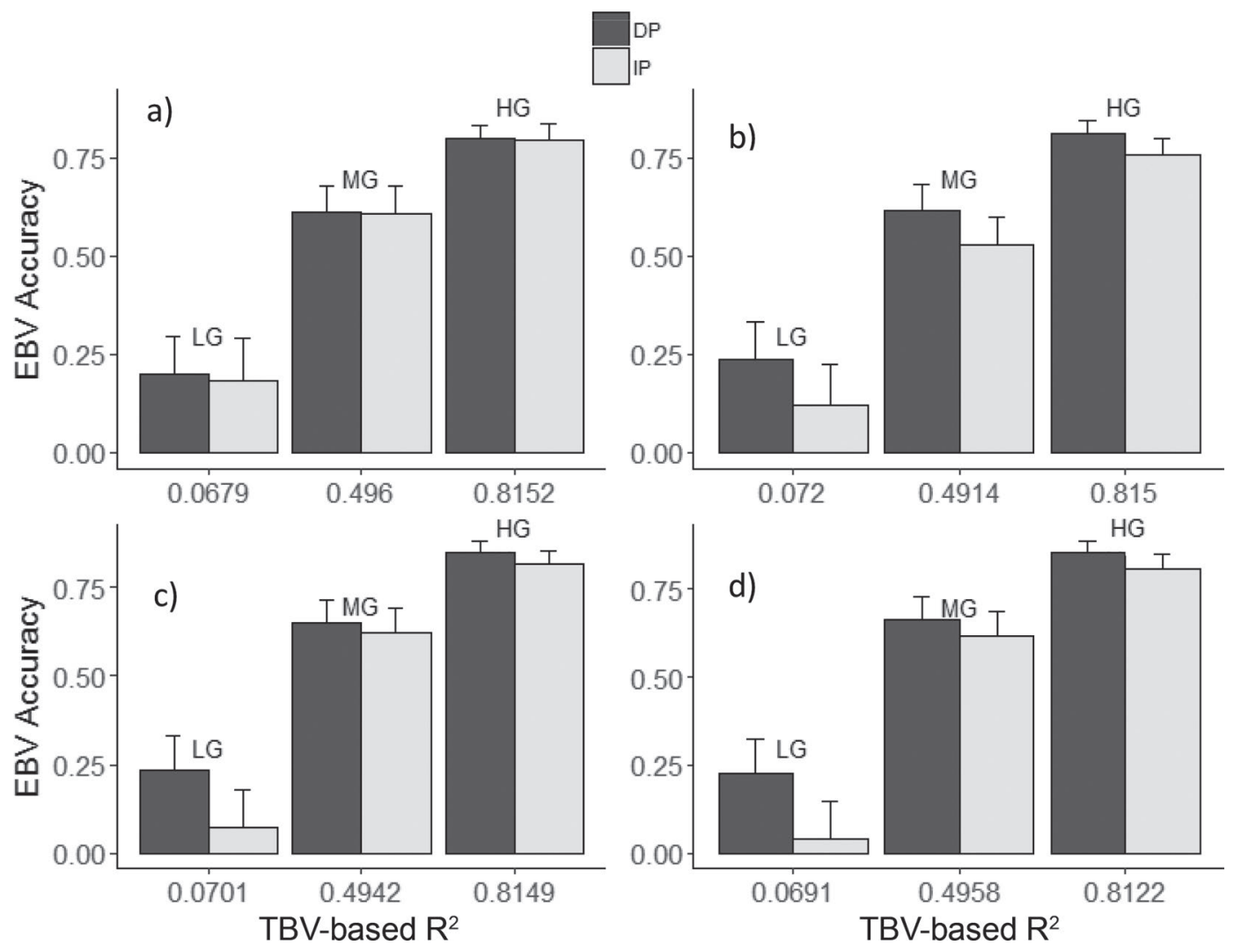

Figure 3. Determination coefficients of calibration models estimated based on true breeding values (TBV) and mean EBV accuracy of sire predicted using the direct (DP) and indirect (IP) prediction approaches. The mean EBV accuracy was predicted under low (LG), medium (MG), and high (HG) genetic correlations with zero (a), low (b), medium (c), and high (d) residual correlation scenarios. The regression coefficient from TBV was used to convert covariance components of traits 2 and 3 into variance components in IP or EBV of traits 2 and 3 into EBV of the intended trait in DP. The error bars represent the SE of prediction. 
Table 4. Mean accuracy ${ }^{1}$ (SE) of predicted phenotypes (corrected for residual effects) of animals with record using the direct (DP) and indirect (IP) prediction approaches under different genetic and residual correlation scenarios using regression coefficients that estimated based on true breeding values $\left(\beta_{\mathrm{g}}\right)$ and true phenotype $\left(\beta_{\mathrm{p}}\right)$

\begin{tabular}{|c|c|c|c|c|c|}
\hline Genetic correlation & Residual correlation & \multicolumn{2}{|c|}{$\mathrm{DP}$} & \multicolumn{2}{|c|}{ IP } \\
\hline \multirow[t]{3}{*}{ Low } & Zero & $0.069(0.038)$ & $0.079(0.034)$ & $0.072(0.039)$ & $0.072(0.040)$ \\
\hline & Medium & $0.290(0.028)$ & $0.140(0.050)$ & $0.314(0.034)$ & $0.312(0.057)$ \\
\hline & High & $0.314(0.024)$ & $0.102(0.051)$ & $0.372(0.035)$ & $0.426(0.073)$ \\
\hline \multirow{2}{*}{ Medium } & Zero & $0.265(0.024)$ & $0.266(0.023)$ & $0.265(0.024)$ & $0.264(0.025)$ \\
\hline & High & $0.483(0.015)$ & $0.479(0.016)$ & $0.640(0.017)$ & $0.667(0.024)$ \\
\hline \multirow[t]{4}{*}{ High } & Zero & $0.345(0.020)$ & $0.346(0.020)$ & $0.345(0.020)$ & $0.344(0.020)$ \\
\hline & Low & $0.339(0.022)$ & $0.336(0.022)$ & $0.366(0.023)$ & $0.372(0.022)$ \\
\hline & Medium & $0.553(0.012)$ & $0.553(0.012)$ & $0.648(0.010)$ & $0.646(0.015)$ \\
\hline & High & $0.565(0.011)$ & $0.565(0.011)$ & $0.739(0.011)$ & $0.742(0.020)$ \\
\hline
\end{tabular}

$\overline{{ }^{1} \text { Accuracy was defined as a correlation between predicted phenotype and simulated true phenotype values (TPV). Average of } 100 \text { replicates per }}$ scenario is reported and the SE were calculated as the SD of the 100 phenotypic accuracies for each scenario.

no significant $(P>0.05)$ difference in performance between using $\beta_{\mathrm{g}}$ and $\beta_{\mathrm{p}}$, except at low genetic correlation with medium or high residual correlation scenarios, where using $\beta_{\mathrm{p}}$ resulted in significantly $(P=0)$ higher accuracies (Table 4). In the IP approach, accuracies of predicted phenotypes were generally slightly higher, but significant $(P<0.01)$ only at low or medium genetic correlations with high residual correlation when using $\beta_{\mathrm{g}}$ than when using $\beta_{\mathrm{p}}$. This is contrary to the EBV accuracy comparison made between using the $\beta_{g}$ and $\beta_{\mathrm{p}}$ within either the DP or IP approach. Similarly, at low genetic correlation, accuracy of phenotypic prediction was significantly $(P=0)$ higher when using $\beta_{\mathrm{p}}$ in DP (Table 4), but accuracy of EBV prediction was significantly $(P=0)$ higher in using the $\beta_{\mathrm{g}}$ in the DP approach (Table 3 ).

\section{Accuracy of $\widehat{P N E}_{I P}$ and $\widehat{P N E}_{D P}$ Versus Accuracy of Calibration Equations}

The relationships between phenotype prediction accuracy of the IP and DP approaches with the predictive ability of calibration models $\left(\mathrm{R}^{2}\right)$ were evaluated and are depicted in Figure 4 when using $\beta_{\mathrm{p}}$ and $\mathrm{R}_{\mathrm{p}}{ }^{2}$ and in Figure 5 in using $\beta_{\mathrm{g}}$ and $\mathrm{R}_{\mathrm{g}}{ }^{2}$. Similar to the accuracy of EBV, phenotypic prediction accuracy increased with an increase in predictive ability of calibration models. Both at zero (Figure 4a) and low (Figure 4b) residual correlation scenarios, predictive ability of calibration model was very low $\left(\mathrm{R}_{\mathrm{p}}{ }^{2}<0.1\right)$, and most increases in the accuracy of phenotypic prediction in the 2 approaches were due to increase in genetic correlations. At medium residual correlation (Figure $4 \mathrm{c}$ ) the $\mathrm{R}_{\mathrm{p}}{ }^{2}$ increased to a maximum of 0.56 , whereas the corresponding value at a high residual correlation (Figure 4d) was 0.79 . The corresponding maximum value in accuracy of phenotype was 0.55 for DP or 0.65 for IP at the medium residual correlation scenario and was 0.56 for DP or 0.74 at the high residual correlation scenario. As indicated above, the IP approach performs better than the DP for predicting the phenotypes, and the difference in prediction accuracy between the 2 approaches becomes clearer as predictive ability of calibration models $\left(\mathrm{R}_{\mathrm{p}}{ }^{2}\right)$ increased (Figure 4).

In using the $\beta_{\mathrm{g}}$, phenotypic prediction accuracy of the IP and DP approaches also increased with increase in predictive ability of the calibration models $\left(\mathrm{R}_{\mathrm{g}}{ }^{2}\right)$. Increases in genetic correlations between traits increased both performance of the 2 approaches and the calibration models regardless of the residual structures (Figure $5)$. At zero residual correlation (Figure 5a), we found no significant $(P>0.05)$ difference in performance between the 2 approaches as the $\mathrm{R}_{\mathrm{g}}{ }^{2}$ increased. However, at the remaining residual correlation scenarios (Figure 5b-d), the IP approach outperformed the DP approach with increase in $\mathrm{R}_{\mathrm{g}}{ }^{2}$ of calibration models, and the highest difference in performance between the 2 approaches was observed at high residual correlation scenario (Figure $5 \mathrm{~d}$ ). Unlike when using $\beta_{\mathrm{p}}$, it is possible to distinguish between performance of the 2 approaches when using $\beta_{\mathrm{g}}$ at low $\mathrm{R}_{\mathrm{g}}{ }^{2}(<0.1)$, where genetic and residual correlations between traits were low.

\section{Accuracy of Predicted Phenotypes $\left(\widehat{P W E}_{I P}\right.$ and $\left.\widehat{P W E}_{D P}\right)$}

In our study, phenotype for the focal trait was predicted in 2 ways: (1) phenotypic prediction obtained 


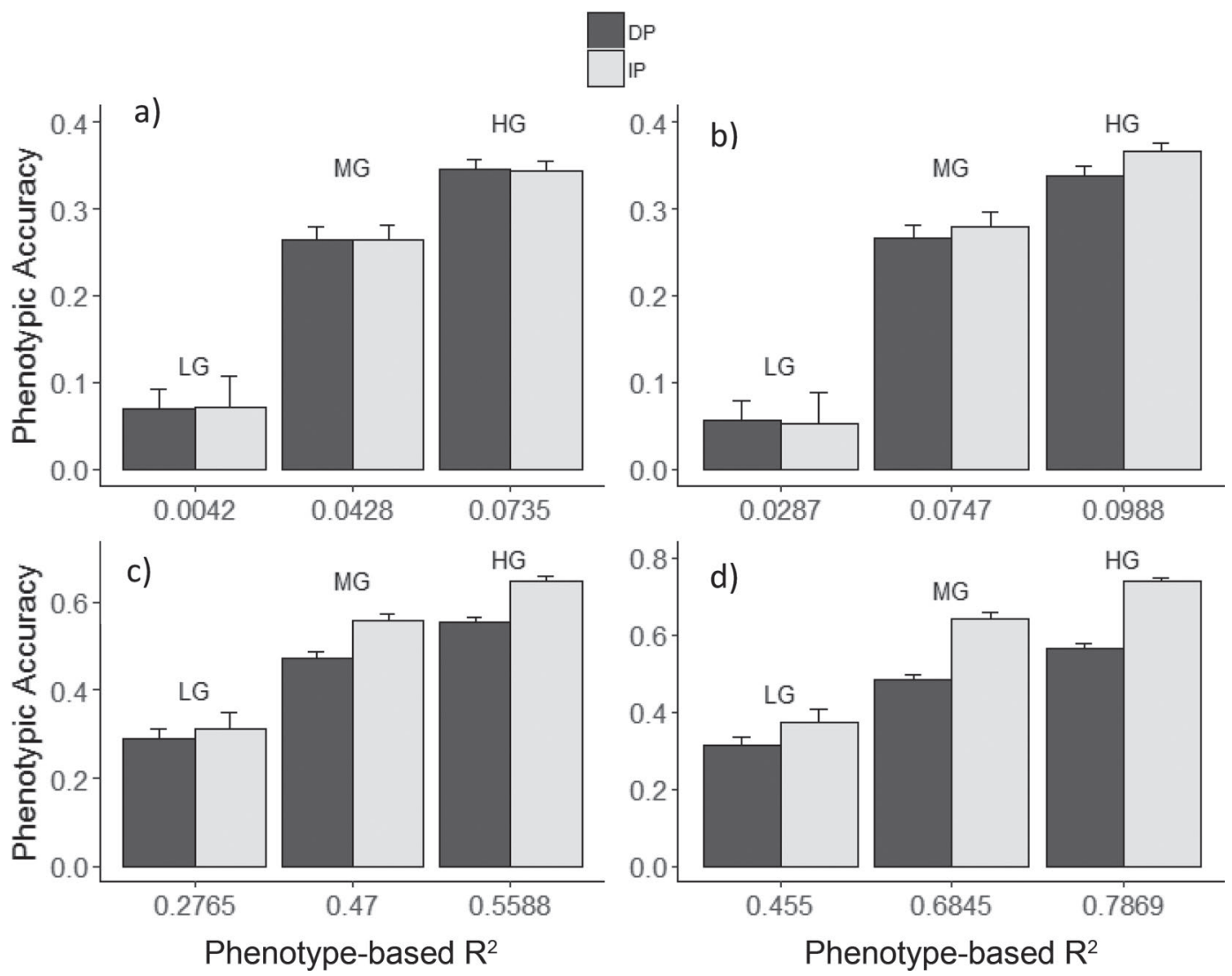

Figure 4. Determination coefficients of calibration models estimated based on phenotype and mean accuracy of predicted phenotypes (without residual effects) using the direct (DP) and indirect (IP) prediction approaches. The mean phenotypic accuracy was predicted under low (LG), medium (MG), and high (HG) genetic correlations with zero (a), low (b), medium (c), and high (d) residual correlation scenarios. The regression coefficient estimated based on true phenotype values was used to convert covariance components of traits 2 and 3 into variance components to be used in IP or predicted phenotypes of traits 2 and 3 into predicted phenotypes of the intended trait in DP. The error bars represent the SE of prediction.

directly from BLUP when such phenotypes did not contain residual effects, and (2) those phenotypes from BLUP and residual effects from BLUP. The former was described in the preceding section whereas the latter phenotypes are presented in this section. In the DP approach, BLUP solutions for residuals of trait 2 and 3 were converted into residuals of the focal trait using $\beta_{\mathrm{r}}$ that were estimates from residual part of simulated phenotypes. This was done assuming that use of appropriate PLS regression coefficients for the different model components would result in better prediction than using only regression coefficient estimated from phenotype. Accordingly, predicted phenotypes of trait 2 and 3 were converted into single-trait predicted phenotypes $\left(\widehat{P N E}_{D P}\right)$ using either $\beta_{\mathrm{p}}$ or $\beta_{\mathrm{g}}$ (as described above), whereas $\beta_{\mathrm{r}}$ was used to convert predicted residual of trait 2 and 3 into single-trait residual $(\hat{e})$. This single-trait residual $(\hat{e})$ was later added to the $\widehat{P N E}_{D P}$ to obtain predicted phenotypes for the focal trait
$\left(\widehat{P W E}_{D P}=\widehat{P N E}_{D P}+\hat{e}\right)$. In the IP approach, predicted phenotypes (i.e., phenotypes corrected for residual effects, $\left.\widehat{P N E}_{I P}\right)$ and residual effects directly obtained from BLUP were added together to generate new predicted phenotypes for the focal trait $\left(\widehat{P W E}_{I P}=\widehat{P N E}_{I P}+\hat{e}\right)$. In addition to the DP- and IPpredicted phenotypes $\left(\widehat{P W E}_{I P}\right.$ and $\left.\widehat{P W E}_{D P}\right)$, phenotypes predicted by classical PLS $\left(\widehat{P}_{P L S}\right)$ were also computed and compared with the $\widehat{P W E}_{I P}$ and $\widehat{P W E}_{D P}$.

Similar to accuracies of phenotypes corrected for residual effects (i.e., $\widehat{P N E}_{I P}$ and $\widehat{P N E}_{D P}$ ), accuracies of phenotypes that contained residual effects (i.e., $\widehat{P W E}_{I P}$ and $\widehat{P W E}_{D P}$ ) increased with an increase in genetic and residual correlations (Table 5). In using $\beta_{\mathrm{p}}$, accuracy of phenotypic prediction in the IP approach was generally similar to the accuracy in the DP approach. This is contrary to accuracy of the $\widehat{P N E}_{I P}$ and $\widehat{P N E}_{D P}$ reported 
in the current (Table 4) and previous (Belay et al., 2017 ) studies. The exceptions were at zero residual correlation with medium or high genetic correlation, where the DP performed significantly $(P<0.05)$ better than the IP approach but with relatively high standard errors. At low residual correlation with medium or high genetic correlation, however, IP performed significantly $(P<0.05)$ better than the DP approach. In using $\beta_{\mathrm{g}}$, accuracy of phenotypic prediction in the DP approach was slightly higher in most scenarios but significantly $(P<0.05)$ higher at zero to low residual correlations with medium or high genetic correlation scenarios than accuracy in the IP approach. In addition, accuracy of phenotypic prediction in the DP approach was significantly $(P<0.05)$ higher when using $\beta_{\mathrm{g}}$ than using $\beta_{\mathrm{p}}$ at zero and low residual correlations. This gives a clue to the importance of using appropriate regression coefficients $\left(\beta_{\mathrm{g}}\right.$ and $\left.\beta_{\mathrm{r}}\right)$ in the DP approach for converting the multitrait to single-trait structure. Within the IP approach, accuracy of phenotypic prediction using $\beta_{\mathrm{p}}$ was equal to the one predicted in using $\beta_{\mathrm{g}}$. These pheno- typic prediction accuracies were also equal to the ones predicted by PLS (results not shown in table), indicating single-trait BLUP analysis (IP) for PLS-predicted traits is not necessary for ultimate prediction of phenotypes.

\section{Accuracy of $\widehat{P W E}_{I P}$ and $\widehat{P W E}_{D P}$ Versus Accuracy of Calibration Equations}

The relationships between accuracy of the $\widehat{P W E}_{I P}$ or $\widehat{P W E}_{D P}$ and the predictive ability of calibration models $\left(\mathrm{R}^{2}\right)$ are depicted in Figure 6 for $\beta_{\mathrm{p}}$ and $\mathrm{R}_{\mathrm{p}}{ }^{2}$ and in Figure 7 for $\beta_{\mathrm{g}}$ and $\mathrm{R}_{\mathrm{g}}{ }^{2}$. When using the $\beta_{\mathrm{p}}$, accuracy of phenotypic prediction increased with an increase in $\mathrm{R}_{\mathrm{p}}{ }^{2}$ (Figure 6); this is similar to accuracy of EBV and $\widehat{P N E}_{I P}$ or $\widehat{P N E}_{D P}$ described in the current study. Differences in performance between the 2 approaches were clearer at low $\mathrm{R}_{\mathrm{p}}{ }^{2}(<0.1$; Figure 6a-b) than at medium to high $\mathrm{R}_{\mathrm{g}}{ }^{2}(0.277-0.787$; Figure $6 \mathrm{c}-\mathrm{d})$. This is contrary to performance of the 2 approaches for predicting ac-

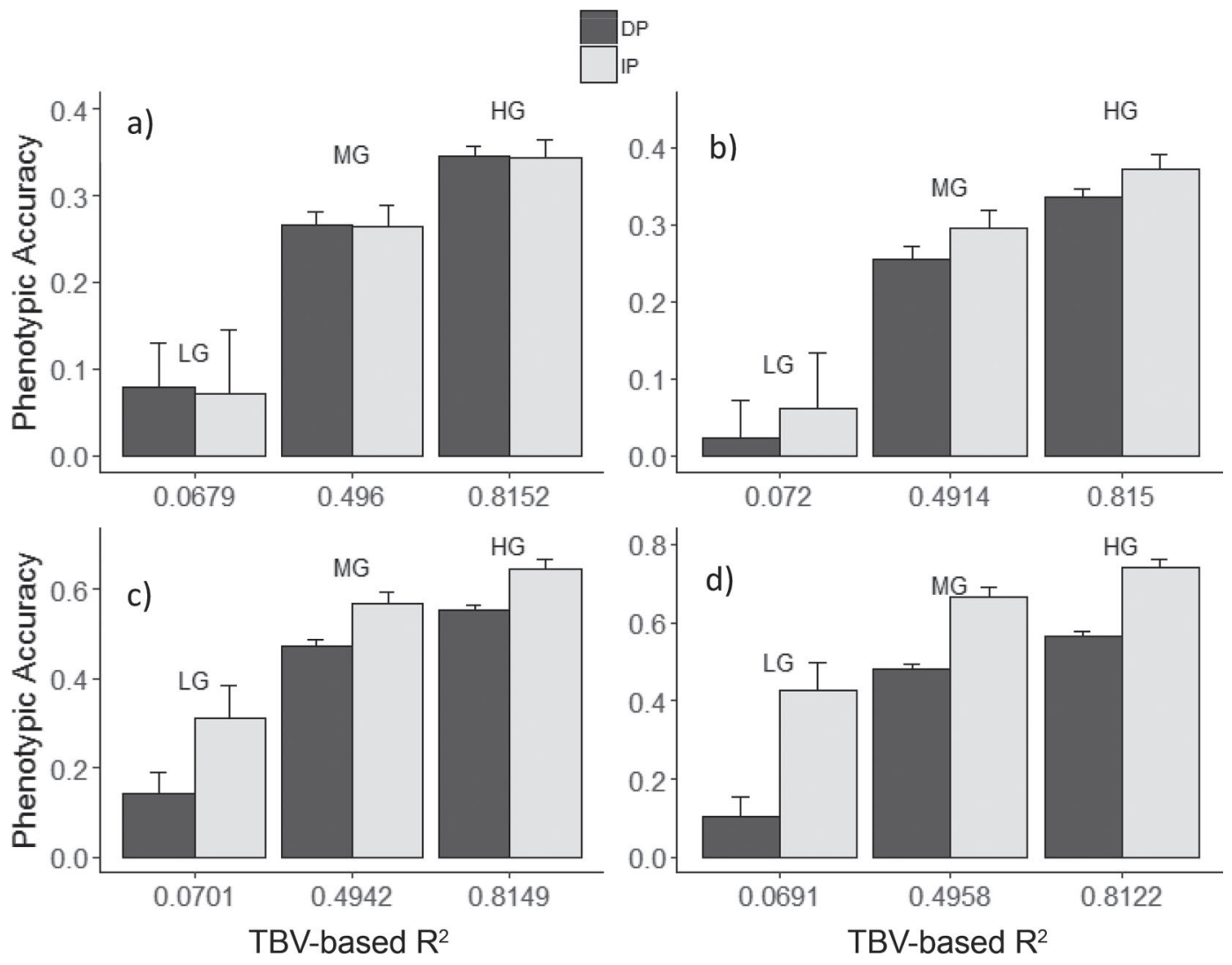

Figure 5. Determination coefficients of calibration models estimated based on true breeding values (TBV) and mean accuracy of predicted phenotypes (without residual effects) using the direct (DP) and indirect (IP) prediction approaches. The mean phenotypic accuracy was predicted under low (LG), medium (MG), and high (HG) genetic correlations with zero (a), low (b), medium (c), and high (d) residual correlation scenarios. The regression coefficient from TBV was used to convert covariance components of traits 2 and 3 into variance components to be used in IP or predicted phenotypes of traits 2 and 3 into predicted phenotypes of the intended trait in DP. The error bars represent the SE of prediction. 

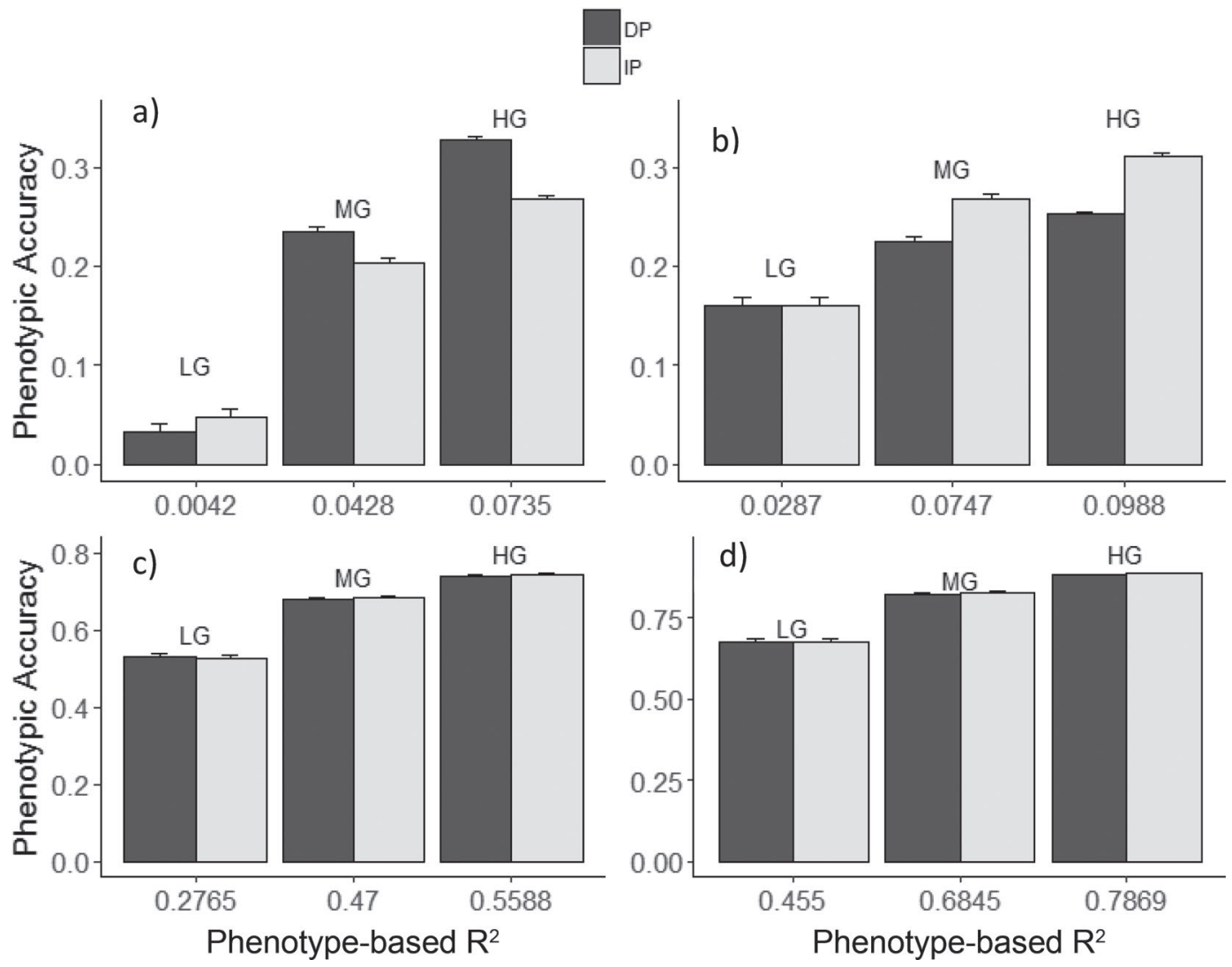

Figure 6. Determination coefficients of calibration models estimated based on phenotypes and mean accuracy of predicted phenotypes (including residual effects) using the direct (DP) and indirect (IP) prediction approaches. The mean phenotypic accuracy was predicted under low (LG), medium (MG), and high (HG) genetic correlations with zero (a), low (b), medium (c), and high (d) residual correlation scenarios. The regression coefficient based on true phenotype values was used to convert covariance components of traits 2 and 3 into variance components to be used in IP or predicted phenotypes of traits 2 and 3 into predicted phenotypes of the intended trait in DP. The error bars represent the SE of prediction.

Table 5. Mean accuracy ${ }^{1}$ (SE) of predicted phenotypic values (including residual effect in the prediction) of animals with records using the direct (DP) and indirect (IP) approaches under different genetic and residual correlation scenarios using regression coefficients that estimated based on true breeding values $\left(\beta_{\mathrm{g}}\right)$ and true phenotypic values $\left(\beta_{\mathrm{p}}\right)$

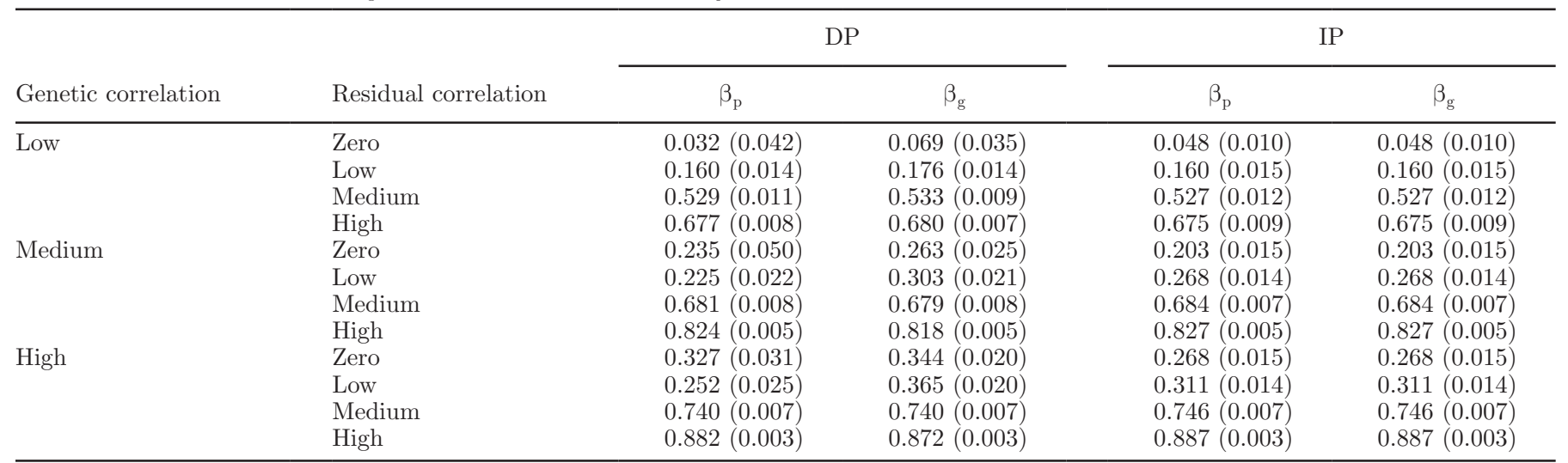

${ }^{1}$ Accuracy was defined as a correlation between predicted phenotype and simulated true phenotype values (TPV). Average of 100 replicates per scenario is reported and the SE were calculated as the SD of the 100 phenotypic accuracies for each scenario. 


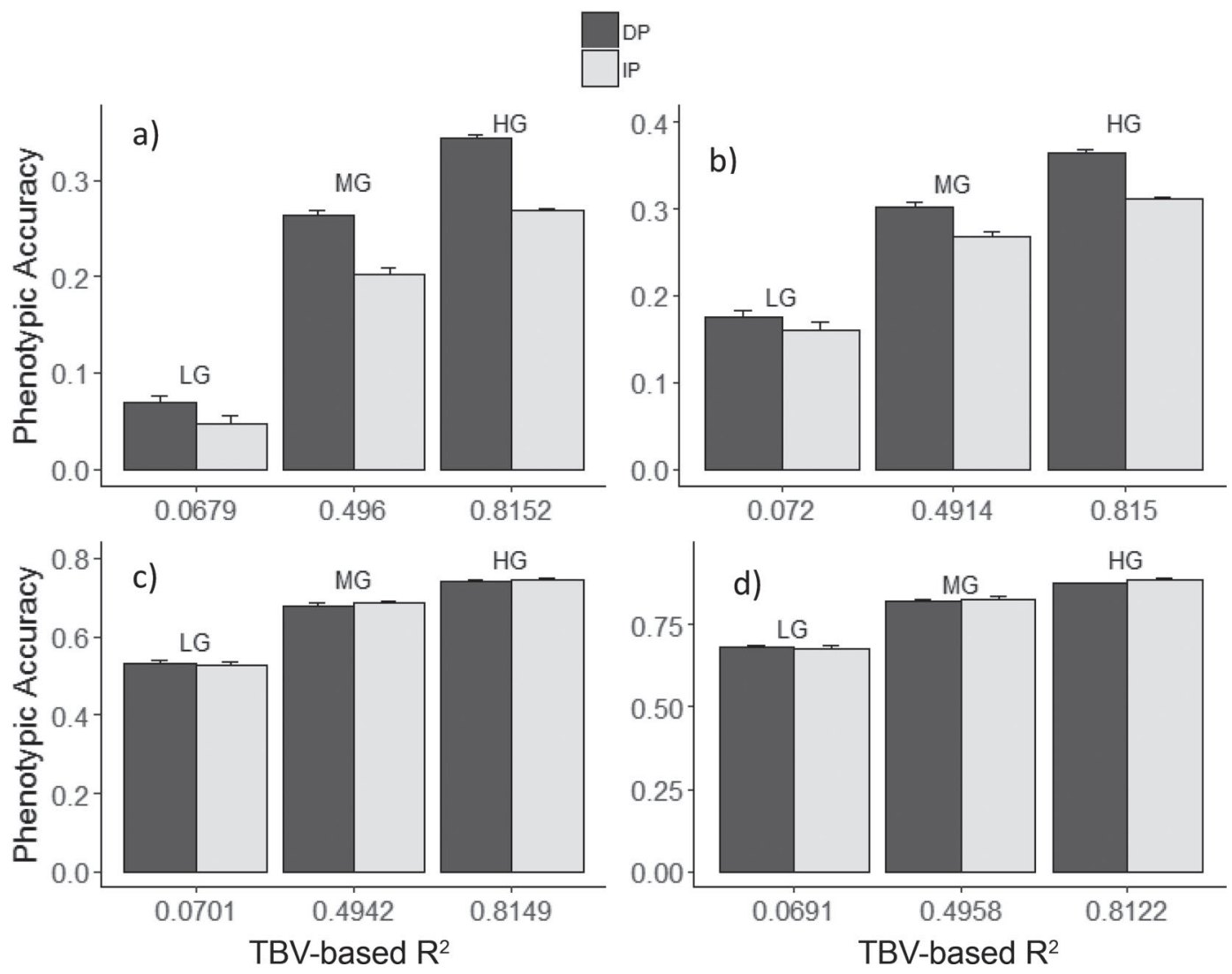

Figure 7. Determination coefficients of calibration models estimated based on true breeding values (TBV) and mean accuracy of predicted phenotypes (including residual effects) using the direct (DP) and indirect (IP) prediction approaches. The mean phenotypic accuracy was predicted under low (LG), medium (MG), and high (HG) genetic correlations with zero (a), low (b), medium (c), and high (d) residual correlation scenarios. The regression coefficient from TBV was used to convert covariance components of traits 2 and 3 into variance components to be used in IP or predicted phenotypes of traits 2 and 3 into predicted phenotypes of the intended trait in DP. The error bars represent the SE of prediction.

curacy of EBV and $\widehat{P N E}_{I P}$ or $\widehat{P N E}_{D P}$, where performance of the 2 approaches overlap at low $\mathrm{R}_{\mathrm{p}}{ }^{2}(<0.1$; Figures 2 and 4).

When using the $\beta_{\mathrm{g}}$, the prediction accuracy of the IP and DP approaches also increased with increase in $\mathrm{R}_{\mathrm{g}}{ }^{2}$ of calibration models (Figure 7). Similar to accuracy of EBV,$\widehat{P N E}_{I P}$, or $\widehat{P N E}_{D P}$, the residual structures had no effect on the $\mathrm{R}_{\mathrm{g}}{ }^{2}$ of calibration models but did have an effect on the accuracy of phenotypic prediction by the 2 approaches (Figure 7). Increase in genetic correlations between traits increased both performance of the 2 approaches and $\mathrm{R}_{\mathrm{g}}{ }^{2}$ of calibration models regardless of the residual structures. At zero (Figure 7a) and low (Figure $7 \mathrm{~b}$ ) residual correlation, differences in performances between the 2 approaches significantly $(P<$ $0.05)$ increased with an increase in $\mathrm{R}_{\mathrm{g}}{ }^{2}$ of calibration models or with an increase in genetic correlations. At medium (Figure 7c) and high (Figure 7d) residual correlation, however, performance of the 2 approaches were similar regardless of increase in $\mathrm{R}_{\mathrm{g}}{ }^{2}$ or in genetic correlations. These results are contrary to performance of the 2 approaches in predicting accuracy for the $\widehat{P N E}_{I P}$ and $\widehat{P N E}_{D P}$ phenotypes (Figures 4 and 5). This indicated that, for traits with low link to predictor variables, better phenotypic prediction would be found with the DP in using both $\beta_{\mathrm{g}}$ and $\beta_{\mathrm{r}}$ together.

\section{CONCLUSIONS}

In this study, performance of the IP and DP approaches under different genetic and residual correlation scenarios were evaluated. In addition, effects of using different regression coefficients $\left(\beta_{\mathrm{g}}, \beta_{\mathrm{p}}\right.$, or $\left.\beta_{\mathrm{r}}\right)$ on accuracy of prediction (EBV and phenotype) were investigated. The relationships between performance of the IP and DP approaches and accuracy of calibration models (for phenotype, genetic, and residual values of the focal trait) were also studied. Accuracies of EBV 
were significantly $(P<0.05)$ higher in the DP approach than in the IP approach, whereas the reverse was true for accuracy of phenotypic prediction (i.e., accuracy of $\left.\widehat{P N E}_{I P}>\widehat{P N E}_{D P}\right)$; the exception was when using $\beta_{\mathrm{g}}$ and $\beta_{\mathrm{r}}$, where accuracy of phenotypic prediction in the DP approach was significantly $(P<0.05)$ higher than that in the IP approach at the zero and low residual correlation scenarios (i.e., accuracy of $\widehat{P W E}_{I P}<\widehat{P W E}_{D P}$ ). Predictive ability of the calibration models increased with improvement in genetic and residual structures between traits. Performance of both IP and DP increased with increase in predictive ability of the calibration models. The exceptions were when using $\beta_{g}$ (where performance of the 2 approaches were not affected by predictive ability of the calibration models at a given genetic scenario) and at low genetic correlation (where accuracy of EBV prediction of the 2 approaches slightly decreases as the $\mathrm{R}_{\mathrm{p}}{ }^{2}$ was increased). Therefore, it is not a good strategy to use the DP approach for phenotypic prediction, except when the $\beta_{\mathrm{g}}$ and $\beta_{\mathrm{r}}$ that are difficult to estimate using real data would be available. Use of the DP approach for prediction of EBV seems useful whereas the IP- or PLS-based prediction equations are a method of choice for phenotypic prediction.

\section{ACKNOWLEDGMENTS}

The Department of Animal and Aquacultural Sciences, Norwegian University of Life Sciences financially supported this study.

\section{REFERENCES}

Belay, T. K., B. S. Dagnachew, Z. M. Kowalski, and T. Ådnøy. 2017. An attempt at predicting blood $\beta$-hydroxybutyrate from Fouriertransform mid-infrared spectra of milk using multivariate mixed models in Polish dairy cattle. J. Dairy Sci. 100:6312-6326.

Belay, T. K., K. Słoniewski, Z. Kowalski, and T. Adnoy. 2015. Predicting ketosis from milk mid infrared (MIR) spectra using multivariate mixed models in Proc. Third DairyCare Conference, Croatia, Zadar. Accessed Mar. 16, 2017. www.dairycareaction.org/uploads/ 2/4/2/6/24266896/belay_tuesday.pdf.

Bittante, G., and A. Cecchinato. 2013. Genetic analysis of the Fouriertransform infrared spectra of bovine milk with emphasis on individual wavelengths related to specific chemical bonds. J. Dairy Sci. 96:5991-6006.

Bonfatti, V., D. Vicario, L. Degano, A. Lugo, and P. Carnier. 2017. Comparison between direct and indirect methods for exploiting Fourier transform spectral information in estimation of breeding values for fine composition and technological properties of milk. J. Dairy Sci. 100:2057-2067.

Dagnachew, B. S., A. Kohler, and T. Ådnøy. 2013a. Genetic and environmental information in goat milk Fourier transform infrared spectra. J. Dairy Sci. 96:3973-3985.

Dagnachew, B. S., T. Meuwissen, and T. Ådnøy. 2013b. Genetic components of milk Fourier-transform infrared spectra used to predict breeding values for milk composition and quality traits in dairy goats. J. Dairy Sci. 96:5933-5942.

Mevik, B.-H., and R. Wehrens. 2007. The pls package: Principal component and partial least squares regression in R. J. Stat. Softw. 18:1-24.

Meyer, K. 2007. WOMBAT - A tool for mixed model analyses in quantitative genetics by restricted maximum likelihood (REML). J. Zhejiang Univ. Sci. B 8:815-821.

R Core Team. (2016). R: A language and environment for statistical computing. R Foundation for Statistical Computing, Vienna, Austria. https://www.R-project.org/.

Soyeurt, H., I. Misztal, and N. Gengler. 2010. Genetic variability of milk components based on mid-infrared spectral data. J. Dairy Sci. 93:1722-1728.

Wang, Q., A. Hulzebosch, and H. Bovenhuis. 2016. Genetic and environmental variation in bovine milk infrared spectra. J. Dairy Sci 99:6793-6803.

\section{APPENDIX: ESTIMATION OF GENETIC REGRESSION COEFFICIENT IN REAL DATA}

Assuming the focal trait is $\mathrm{F}$ and the 2 spectra variables (latent traits) are L1 and L2. In the simulation study, we obtained $\beta_{\mathrm{g}}$ (genetic regression coefficient) by fitting a regression of true breeding values (TBV) of the focal trait on the TBV of the 2 latent traits. This can be written as

$$
T B V_{F}=\mu+\beta_{\mathrm{g}_{L 1}} \cdot T B V_{L 1}+\beta_{\mathrm{g}_{L 2}} \cdot T B V_{L 2}+e
$$

where $e$ can be zero. Any of the genetic regression coefficient $\left(\beta_{\mathrm{g}_{L 1}}\right.$ or $\left.\beta_{\mathrm{g}_{L 2}}\right)$ is estimated as

$$
\beta_{\mathrm{g}_{L}}=\frac{\operatorname{cov}\left(T B V_{F}, T B V_{L}\right)}{\sigma_{T B V_{L}}^{2}} .
$$

Because this was a simulation study, $\beta_{\mathrm{g}}$ was obtained from the TBV that were simulated from all the 2 traits.
However, in a real data setting, an estimate of the genetic regression coefficient $\left(\widehat{\beta}_{\mathrm{g}}\right)$ can be obtained through a series of steps.

First, L1 and L2 are directly available, as they are spectra data (this can and will be available for large group of animals). And second, the focal trait $\left(y_{F}\right)$ can be (is) measured and used to obtain the prediction equation. Thus, with these 2 information sources, we can

1. Estimate variance component $\left(\sigma_{a_{L}}^{2}\right)$ and predict breeding values for each of the spectral variables (i.e., $E B V_{\mathrm{L} 1}, E B V_{\mathrm{L} 2}$, and so on) with a singletrait animal model or for latent traits with a multitrait model and back-transforming EBV of the latent traits into EBV of spectral variables; and 
2. Compute the genetic regression coefficient $\left(\hat{\beta}_{g_{L}}\right)$ for $E B V_{F}$ on each spectra variable as

$$
\hat{\beta}_{\mathrm{g}_{L}}=\frac{\operatorname{cov}\left(y_{F}, E B V_{L}\right)}{\sigma_{a_{L}}^{2}} .
$$

This can be extended as

$$
\begin{gathered}
\hat{\beta}_{\mathrm{g}_{L}}=\frac{\operatorname{cov}\left(E B V_{F}+R_{F}, E B V_{L}\right)}{\sigma_{a_{L}}^{2}}= \\
\frac{\operatorname{cov}\left(E B V_{F}, E B V_{L}\right)+\operatorname{cov}\left(R_{F}, E B V_{L}\right)}{\sigma_{a_{L}}^{2}}
\end{gathered}
$$

but because $\operatorname{cov}\left(R_{F}, E B V_{L}\right)=0$, then $\hat{\beta}_{\mathrm{g}_{L}}$ is of only the genetic covariance between $F$ and $L$,

$$
\hat{\beta}_{g_{L}}=\frac{\operatorname{cov}\left(E B V_{F}, E B V_{L}\right)}{\sigma_{a_{L}}^{2}} .
$$

It is important to note that instead of using $\sigma_{E B V_{L}}^{2}$ as the denominator, we have opted to use $\sigma_{a_{L}}^{2}$. This is because if we want $\hat{\beta}_{\mathrm{g}_{L}}$ to approximate $\beta_{\mathrm{g}_{L}}$, as in our simulations, then the appropriate variance parameter to use will be $\sigma_{a_{L}}^{2}$. We know that $\sigma_{E B V_{L}}^{2}=r \cdot \sigma_{a_{L}}^{2}$, with $r$ being the accuracy of the EBV. To obtain a confidence interval for the genetic regression coefficient, a cross validation scheme can be implemented by not using all data to compute the $\hat{\beta}_{\mathrm{g}_{L}}$, but a random sample for several repeated samplings. 